\title{
Time Discounting: Psychophysics of Intertemporal and Probabilistic Choices
}

\author{
Taiki Takahashi $^{\mathrm{a}}$, Ruokang $\operatorname{Han}^{\mathrm{b}}$, and Fumihiko Nakamura ${ }^{\mathrm{c}}$
}

\begin{abstract}
Intertemporal and probabilistic choices have been attracting attention in behavioral neuroeconomics. Although recent advances in neuroeconomics demonstrated neural processes underlying intertemporal and probabilistic choices, the roles of psychophysical effects on the choices have largely been understudied. In this paper, we review the roles of psychophysical effects in time and risk attitudes. It is shown that Loewenstein-Prelec's generalized hyperbolic temporal discount function (Loewenstein and Prelec (1992)) and Prelec's probability weighting function (Prelec (1998)) are naturally derived from psychophysical laws of time-perception.
\end{abstract}

Keywords: Intertemporal choice, risk, discounting, psychophysics, neuroeconomics JEL Classification Numbers: D90, D03, D87

\section{Introduction}

People devalue delayed and/or uncertain rewards, which correspond to temporal discounting and risk aversion. In economics, the degree to which a delayed reward is discounted is parametrized with time preference (a time-discount rate); while the degree to which an uncertain reward is discounted is parametrized with risk attitude. Furthermore, neoclassical economics has been assuming dynamic consistency (Samuelson (1937)) and the independence axiom (von Neumann and Morgenstern (1947)) for decision over time and under uncertainty, respectively, by assuming the rationality in people's decision making. However, subsequent empirical studies demonstrated that people are dynamically inconsistent in intertemporal choice (Loewenstein and Prelec (1992), Frederick et al. (2002)) and violate the independence axiom in decision under risk (Allais (1953), Kahneman and Tversky (1979)). Therefore, recent studies in neuroeconomics have tried to answer why the observed "irrationality" (anomaly) occurs by examining the neural processes underlying intertemporal (McClure et al. (2004), Kable and Glimcher (2007)) and probabilistic choices (Paulus and Frank (2006)). How-

\footnotetext{
* The research reported in this paper was supported by a grant from the Grant- in-Aid for Scientific Research (Global COE) from the Ministry of Education, Culture, Sports, Science and Technology of Japan.

a Department of Behavioral Science, Center for Experimental Research in Social Sciences, Hokkaido University e-mail: taikitakahashi@gmail.com

${ }^{\mathrm{b}}$ Department of Behavioral Science, Center for Experimental Research in Social Sciences, Hokkaido University e-mail: hanruokang@gmail.com

${ }^{c}$ Department of Behavioral Science, Center for Experimental Research in Social Sciences, Hokkaido University e-mail: bunhikonakamura@gmail.com
}

ever, partly because the present neuroimaging techniques are not quantitatively accurate enough, the accounts of the observed "irrational" (anomalous) tendencies have been unclear. Therefore, it is necessary to utilize quantitative mathematical frameworks into behavioral neuroeconomics, in order to account for the irrational behaviors ("anomalies"). In this paper, we show that incorporating the effects of psychophysical laws into economic models of decisions over time and uncertainty may provide the parsimonious accounts of the violations of dynamic consistency and the independence axiom, in a quantitatively rigorous manner.

This paper is organized as follows. In section 2, intertemporal choice models are briefly introduced and empirical findings in behavioral neuroeconomics on irraltional intertemporal choice are addressed. In section 3 , economic theories of decision under risk are explained and how behavioral economic theory (i.e., non-expected utility theories such as prospect theory) can capture the irrationality in decision under risk is addressed. In section 3, we address how psychophysics of timeperception can account for the irrationality in intertemporal choice. Furthermore, we extend the theory of psychophysics of time-perception into decision under risk and demonstrate this theory can also explain the violation of the independence axiom. Finally, in section 4, some implications from the presently reviewed findings for future studies in behavioral neuroeconomics are stated.

\section{Intertemporal choice models in behavio- ral neuroeconomics}

\section{1. Exponential discounting}

Dynamically consistent temporal discounting (simple exponential discounting), which is typically assumed in neoclassical economic theory (Samuelson (1937)) follows 
the exponential equation:

$$
V(D)=V(0) \exp (-k D)
$$

where $\mathrm{V}$ is the subjective value of a reward which can be received at delay $\mathrm{D}$ and, importantly, $\mathrm{D}$ is the objective (physical) time-duration of delay until the delivery of reward. The free parameter $k$ is an index of the degree to which a subject discounts the delayed reward, i.e., larger $k$ values correspond to more rapid (impatient) delay discounting. In simple exponential discounting, there is no dynamic inconsistency in intertemporal choice, because a discount rate: $=-(d V(D) / d D) / V=k$ is independent of $\mathrm{D}$ (a time-constant discount rate). However, empirical examinations of intertemporal choice demonstrated that people do not behave in a dynamically consistent manner.

\section{2. Impulsivity and inconsistency in intertem- poral choice}

Impulsivity (impatience) in intertemporal choice is defined as strong preference for small immediate rewards over large delayed ones. For instance, subject A who prefers "one apple available one year later" to "two apples available one year and a week later" is more impulsive than subject B who prefers "two apples available one year and a week later" to "one apple available one year later" (most people may behave as the subject B, in this example). In neoclassical economic theory, as stated, it has been assumed that subjects have dynamically consistent intertemporal choice behavior (Samuelson (1937)).

However, recent behavioral and neuroeconomic evidence indicates that human and animal discounting rates are changeable, more specifically, decreasing towards the future (decreasing impatience), resulting in preference reversal over time (Loewenstein and Prelec, (1992), Frederick et al. (2002)). Suppose the above example again. If the same subject B prefers "one apple available today" to "two apples available one week later" (again, most people may make decisions in this way), her intertemporal choices are reversed although delays (time-intervals) from the sooner rewards to later ones are the same (i.e., a week) in the two intertemporal choice problems ("dynamic inconsistency"). As shown in the examples, if subjects prefer larger later rewards in intertemporal choice in the distant future, but prefer smaller sooner rewards in intertemporal choice in the near future, their intertemproal choices are dynamically inconsistent, because their preferences reverse as time passes. On the contrary, if someone prefers smaller sooner rewards in both examples; i.e. "one apple available one year later" and "one apple available today", her intertemporal choice is impulsive but dynamically consistent, because preference does not reverse over time. In behavioral economics, several intertemporal choice models have been proposed in order to describe actual human behavior in intertemporal decision-making.

\section{3. Intertemporal choice models in behavioral neuroeconomics}

\section{3. 1. Simple hyperbolic discounting}

Most studies in behavioral neuroeconomics and psychopharmacology have been utilizing a simple form of the hyperbola-like discount model (a simple hyperbolic discount function) (Ainslie (2001), Frederick et al. (2002)):

$$
V=V(0) /\left(1+k_{s} D\right)
$$

where $k_{s}$ is a simple hyperbolic discount rate, $\mathrm{V}(\mathrm{D})$ is again a discounted utility of the delayed reward obtained at objective delay D. In the simple hyperbolic discounting, a time-discount rate $=-(d V(D) / d D) / V=k_{s} /\left(1+k_{s}\right.$ $D)$, which is also a decreasing function of delay (decreasing impatience) when $k_{s}>0$ (i.e. "positive time preference"), resulting in preference reversal over time as shown in section 2.2. It is to be noted that, in behavioral psychology, the simple hyperbolic function has been proposed based on "matching law" (Rachlin (2006)).

\section{3. 2. Generalized hyperbolic discounting and q- exponential discounting}

In behavioral economics, Loewenstein and Prelec proposed the following generalized hyperbolic discount function:

$$
V=V(0) /(1+x D)^{y / x},
$$

where $\mathrm{x}$ and $\mathrm{y}$ are positive real free parameters. Note that $\mathrm{x}$ determines how much the generalized hyperbolic function departs from consistent temporal discounting; i. e., when $\mathrm{x}$ approaches to zero, the generalized hyperbolic function approaches to the exponential discount function: $\mathrm{V}=\mathrm{V}(0) \exp (-\mathrm{yD})$. Also, when $\mathrm{y}=\mathrm{x}$, Equation (3) is equivalent to the simple hyperbolic function (Equation (2)). It is further to be noted that the generalized hyperbolic discount function satisfies the matching law in behavioral psychology (Loewenstein and Prelec, 1992).

Recent studies in econophysics have proposed and examined the following q-exponential discount function based on Tsallis' statistics (Cajueiro (2006), Takahashi (2007) and Takahashi et al. (2007)):

$$
V_{q}(D)=V_{q}(0) / \exp \left(k_{q} D\right)=V_{q}(0) /\left[1+(1-q) k_{q} D\right]^{1 /(1-q)},
$$

where $\exp _{q}()$ is a q-exponential function, which has been developed in Tsallis' non-extensive thermostatistics (Tsallis 1988), and $\mathrm{q}$ and $\mathrm{k}_{\mathrm{q}}$ are free parameters. The q-exponential discount function is mathematically equivalent to Loewenstein and Prelec's generalized hyperbolic function. Moreover, parameter q indicates the deviation from exponential discounting; when $q=0$, the q-exponential discounting is equivalent to the simple hyperbolic function (Equation (2)) and when $q \rightarrow 1$, the qexponential discounting is equivalent to the exponential function (Equation (1)). The advantage of the q-exponential function (Equation (4)) over the original Loewen- 
stein-Prelec's generalized hyperbolic function is that the q-exponential function can parametrize the deviation of temporal discounting from an exponential function as $1-q$. A recent study demonstrated that the q-exponential discount model better explained human intertemporal choice behavior than the exponential and simple hyperbolic discount models (Takahashi et al. (2008)) even considering the model complexity.

\section{Decision under risk}

In game theory and microeconomics, an expected utility theory (von Neumann and Morgenstern (1947)) has been utilized. Von Neumann and Morgenstern's expected utility theory has been constructed upon the assumption of human rationality in decision under risk: i.e., the independence axiom. A French economist Allais (1953) criticized the assumption and suggested the violation of the independence axiom in human decision making under risk. Further studies in behavioral economics by Kahneman and Tversky (1979) formulated a prospect theory in order to describe actual human decision under risk including the violation of the independence axiom. In their seminal paper on prospect theory, Kahneman and Tversky (1979) explained the observed violations of rationality in decision under risk by introducing a nonlinear transformation of objective probabilities into "decision weights" $p \rightarrow w(p)$ for $\mathrm{p}, w(p)$ $\in[0,1]$, where $p$ is the probability of winning a reward and $\mathrm{w}(\mathrm{p})$ is the probability weighting function determining the decision weight for the probability and satisfying $w(0)=0$ and $w(1)=1$. Subsequently, a behavioral economist Prelec derived, from several preference axioms, the probability weighting function:

$$
w(p)=\exp \left(-(-\ln p)^{\alpha}\right)
$$

where $\alpha \in[0,1]$ is a free parameter determining the nonlinear distortion of the subjective probability in decision under risk (Prelec (1998)). Note that Prelec's probability weighting function satisfies $\mathrm{w}(0)=0, \mathrm{w}(1 / \mathrm{e})=1 / \mathrm{e}$ and $\mathrm{w}$ $(1)=1$ (i.e., $1 / \mathrm{e}$ is a fixed point).

In Kahneman-Tversky's prospect theory, the subjective value of the uncertain reward $V(x, p)$ is

$$
V(x, p)=v(x) w(p)
$$

where $\mathrm{v}(\mathrm{x})$ is the value of the reward $\mathrm{x}$, and $\mathrm{p}$ is the probability of winning the reward. It can be stated that, in expected utility theory, $\mathrm{w}(\mathrm{p})=\mathrm{p}$ (i.e., linear probability weighting) is assumed; in other words, KahnemanTversky's prospect theory accounts for the violation of the von Neumann-Morgenstern's independence axiom by introducing a nonlinear perception/weighting of probability values in decision under risk.

\section{Psychophysical theory of intertemporal and probabilistic choices}

\section{1. Psychophysics of time-perception and inter- temporal choice}

Studies in theoretical neuroeconomics proposed that combining psychophysical effects on time-perception such as Weber-Fechner law (i.e. logarithmic time-perception) with the exponential discounting may be capable of describing anomalies in empirically observed intertemporal choice behavior such as decreasing impatience and preference reversal (Takahashi $(2005,2006))$. Let us now suppose time-perception follows logarithmic function (Weber-Fechner law). Because in logarithmic time-perception, subjective time-duration $\tau$ (psychological time) is expressed as:

$$
\tau=a \ln (1+b D)
$$

Exponential discounting with the subjective delay $\tau$ (exponential discounting with logarithmic time-perception) is formulated as:

$$
\begin{aligned}
& V(D)=V(0) \exp (-k \tau)=V(0) \exp (-k a \ln (1+b D)) \\
& \quad=V(0) /(1+b D)^{g},
\end{aligned}
$$

where $\mathrm{D}$ is an objective/physical delay length and $\mathrm{b}$ and $g$ $=k a$ are free parameters. It should be noted that Equation (8) is the same as a general hyperbolic discount function (Equation (3)) and the q-exponential function (Equation (4)). In Equation (8), a discount rate: $=-(d V$ (D) $/ d D) / V=b g /(1+b D)$ is a decreasing function of delay (decreasing impatience) when $b$ and $g$ are larger than zero, resulting in preference reversal over time. In this manner, by incorporating the psychophysical effect of nonlinear time-perception on intertemporal choice into mathematical models of intertemporal choice, we can account for the reported anomaly in intertemporal choice (i.e., decreasing impatience and preference reversal over time). Recent behavioral economic studies experimentally confirmed this account of dynamically inconsistent intertemporal choice (Zauberman et al. (2009), Kim and Zauberman (2009)), by directly measuring people's time-perception. It is also to be noted that Kinari et al. (2009) demonstrated that a parameter-free logarithmic time-perception function did not better account for human temporal discounting, indicating that the inclusion of free parameters in time-perception function is necessary.

\section{2. Psychophysics of waiting-time perception and decision under risk}

Rachlin and colleagues proposed that delay until receipt of gains in intertemporal choice and uncertainty of winning of gains in probabilistic choice may be equivalent (Rachlin et al. (1991)). In this theory, a decrease in a probability of winning an uncertain reward psychologically corresponds to an increase in a delay until winning the reward. Specifically, an average waiting time until winning an uncertain reward is propor- 
tional to an odds against: $(1 / p)-1\left(:=\mathrm{O}_{\mathrm{p}}\right)$, where $p$ is a probability of winning the uncertain reward (note that in this theory, the agent spontaneously assumes that the system is ergodic). Therefore, decision-making models in intertemporal choice can straightforwardly be extended into probabilistic choice, by replacing a parameter of delay in intertemporal choice models with the odds against parameter. Therefore, it can be expected that the psychophysical effects of nonlinear waiting-time perception can also account for the nonlinear proability weighting in Kahneman-Tversky's prospect theory.

This extension has been performed by Takahashi (2011). We can start from a natural assumption that "subjective waiting time in probabilistic choices" $\left(:=\tau_{\mathrm{p}}\right)$ is also logarithmic in "physical waiting time" $\left(:=D_{p}\right)$ which is proportional to "odds against in terms of physical probability" $\left(=\mathrm{O}_{\mathrm{p}}\right)$ :

$$
\tau_{p}\left(O_{p}\right)=a_{p} \ln \left(1+b_{p} t_{p} O_{p}\right)=a_{p} \ln \left[1+b_{p} t_{p}(1 / p-1)\right]
$$

where $a_{p}$ and $b_{p}$ are positive free parameters, and $t_{p}$ is the average time-interval between trials in repeated gambles (i.e., delay $\mathrm{D}_{\mathrm{p}}=\mathrm{t}_{\mathrm{p}} \mathrm{O}_{\mathrm{p}}$, referred to as "physical waiting time"). Then, let us further assume that "subjective waiting time in terms of subjective probability (i.e., the probability weighting function)" (:= $\left.\tau_{\mathrm{w}}\right)$ is also logarithmic in "odds against in terms of the probability weighting function" $\left(:=\mathrm{O}_{\mathrm{w}}\right)$ :

$$
\begin{aligned}
& \tau_{w}\left(O_{w}\right)=a_{w} \ln \left(1+b_{w} t_{w} O_{w}\right) \\
& =a_{w} \ln \left[1+b_{w} t_{w}(1 / w(p)-1)\right],
\end{aligned}
$$

where $a_{w}$ and $b_{w}$ are positive free parameters, and $t_{w}$ is the average inter-trial time of probabilistic choices in terms of probability weighting w(p) (i.e., delay in terms of w(p) is $D_{w}=t_{w} O_{w}$, referred to as "probability-weighted waiting time").

In order to examine the relation between the probability weighting function and a physical probability, we now suppose that a mathematical relation between perceived "physical waiting time" and perceived "probability-weighted waiting time" follows Stevens' power law (in temporal discounting literature, this psychophysical law was introduced by Takahashi (2006) in order to account for "subadditivity" of the time-discount factor):

$$
\tau_{w}\left(O_{w}\right)=\tau_{p}\left(O_{p}\right)^{s}(s \in[0,1]) .
$$

In Stevens' power law, the perception and stimulus are related by a power law, also known as the third psychophysical law, which was revealed with a magnitude estimation method in psychophysics (Stevens (1957)).

Equivalently to Equation (11), we have

$$
a_{w} \ln \left[1+b_{w} t_{w}(1 / w(p)-1)\right]=\left[a_{p} \ln \left[1+b_{p} t_{p}(1 / p-1)\right]\right]^{s} .
$$

By solving Equation (12) in terms of w(p), we obtain

$$
\begin{aligned}
& w(p)= \\
& \exp \left[a_{w}{ }^{-1} a_{p}^{s}\left\{\ln \left[1+b_{p} t_{p}\left(p^{-1}-1\right)\right]\right\}^{s}\right]+b_{w} t_{w}-1 .
\end{aligned}
$$

Here, we can see that $w(0)=0$ and $w(1)=1$, indicating that $\mathrm{w}(\mathrm{p})$ in Equation (13) is the generalized probability weighting function for Kahneman-Tversky's prospect theory.

In order to demonstrate that Prelec's probability weighting function is the special case of this generalized probability weighting function, we then assume that $a_{w}{ }^{-1} a_{p}{ }^{s}=b_{p} t_{p}=b_{w} t_{w}=1$. Consequently, we obtain

$$
w(p)=\exp \left(-(-\ln p)^{s}\right)
$$

which is equivalent to Equation (5) (i.e., Prelec's probability weighting function, Equation (5)). Therefore, the nonlinearity parameter $\alpha$ in Prelec's probability weighting function is an exponent of Stevens' power law of phychophysics of waiting time.

\section{Conclusions and implications for future studies}

As shown in section 3, anomalies in decisions over time and risk are both accounted for by psychophysical laws of time-perception in a unified manner. It is noteworthy that psychophysics of the perception of delay until receipt is shown to play a pivotal role in intertemporal choice; while psychophysics of the perception of waiting time in repeated gambles plays a major role in decision under risk. In behavioral psychology, Rachlin et al. (1991) reported that temporal discounting and risk aversion (defined as aversion to delay until winning in repeated gambles) are correlated, supporting our currently presented theoretical framework. Also, Kim and Zauberman (2009) demonstrated that individual differences in temporal discounting are related to individual differences in anticipatory time-perception in temporal discounting tasks. However, no study to date examined the role of subjective waiting time in repeated gambles in risk preference. This point should be examined in future behavioral economics. Taken together, future studies in behavioral economics should examine whether common psychological processes mediate anomalies in both time and risk preferences. Moreover, future neuroeconomic studies should examine neural substrates underlying these psychophysical effects on temporal discounting and decision under risk.

\section{REFERENCES}

Ainslie, G., 2001. Breakdown of Will. Cambridge University Press, Cambridge, UK.

Allais, M., 1953. Le comportement de l'homme rationnel devant le risque: Critique des postulats et axiomes de l'école Américaine. Econometrica 21, 503-546.

Cajueiro, D. O., 2006. A note on the relevance of the q-expo- 
nential function in the context of intertemporal choices. Physica A 364, 385-388.

Frederick, S., G. Loewenstein, and T. O'Donoghue, 2002. Time discounting and time preference: A critical review. Journal of Economic Literature 40, 350-401.

Kable, J. W. and P. W. Glimcher, 2007. The neural correlates of subjective value during intertemporal choice. Nature Neuroscience 10, 1625-1633.

Kahneman D. and A. Tversky, 1979. Prospect theory an analysis of decision under risk. Economitrica 47, 263-291.

Kim, B. K. and G. Zauberman, 2009. Perception of anticipatory time in temporal discounting. Journal of Neuroscience, Psychology, and Economics 2 (2), 91-101.

Kinari, Y., F. Ohtake, and Y. Tsutsui, 2009 Time discounting, declining impatience and interval effect. Journal of Risk and Uncertainty 39, 87-112.

Loewenstein, G. and D. Prelec, 1992. Anomalies in intertemporal choice: Evidence and an interpretation. The Quarterly Journal of Economics 107, 573-597.

McClure, S. M., D. I. Laibson, G. Loewenstein, and J. D. Cohen, 2004. Separate neural systems value immediate and delayed monetary rewards. Science 306, 503-507.

Paulus, M. P. and L. R. Frank, 2006. Anterior cingulate activity modulates nonlinear decision weight function of uncertain prospects. Neuroimage $30,668^{-77}$.

Prelec, D., 1998. The probability weighting function. Econometrica $66,497-527$.

Rachlin, H., A. Raineri, and D. Cross, 1991. Subjective probability and delay. Jounal of Experimental Analysis of Behavior 55 (2), 233-244.

Rachlin, H., 2006. Notes on discounting. Journal of The Ex- perimental Analysis of Behavior 85, 425-435.

Samuelson, P. A., 1937. A Note on measurement of utility. The Review of Economic Studies 4, 155-161.

Stevens, S. S., 1957. On the psychological law. Psychological Review 64 (3), 153-181.

Takahashi, T., 2011. Psychophysics of the probability weighting function. Physica A 390, 902-905.

Takahashi, T., 2005. Loss of self-control in intertemporal choice may be attributable to logarithmic time-perception. Medical Hypotheses 65, 691-693.

Takahashi, T., 2006. Time-estimation error following WeberFechner law may explain subadditive time-discounting. Medical Hypotheses 67, 1372-1374.

Takahashi, T., 2007. A comparison of intertemporal choices for oneself versus someone else based on Tsallis' statistics. Physica A 385, 637-644.

Takahashi, T., H. Oono, and M. H. Radford, 2007. Empirical estimation of consistency parameter in intertemporal choice based on Tsallis' statistics. Physica A 381, 338-342.

Takahashi, T., H. Oono, and M. H. B. Radford, 2008. Psychophysics of time perception and intertemporal choice models. Physica A 87, 2066-2074.

Tsallis, C., 1988. Possible generalization of Boltzmann-Gibbs entropy. Journal of Statistical Physics 52, 479.

von Neumann, J. and O. Morgenstern, 1947. Theory of Games and Economic Behavior. 2nd ed. Princeton University Press, Princeton, US.

Zauberman, G., B. K. Kim, S. Malkoc, and J. R. Bettman, 2009. Discounting time and time discounting: Subjective time perception and intertemporal preferences. Journal of Marketing Research 46 (4), 543-556. 